\title{
Effect of chemotherapy and radiotherapy on cognitive impairment in colorectal cancer: evidence from Korean National Health Insurance Database Cohort
}

\author{
Kwanghyun Kim ${ }^{1,2}$, Chang Woo Kim³, Aesun Shin ${ }^{4}$, Hyunseok Kang ${ }^{5}$, Sun Jae Jung 1,2 \\ ${ }^{1}$ Department of Preventive Medicine, Yonsei University College of Medicine, Seoul, Korea; ${ }^{2}$ Department of Public Health, Yonsei University, \\ Seoul, Korea; ${ }^{3}$ Department of Surgery, Ajou University School of Medicine, Suwon, Korea; ${ }^{4}$ Department of Preventive Medicine, Seoul National \\ University College of Medicine, Seoul, Korea; ${ }^{5}$ Department of Medicine, University of California, San Francisco, CA, USA
}

\begin{abstract}
OBJECTIVES: We investigated the risk of chemotherapy-related and radiotherapy-related cognitive impairment in colorectal cancer patients.

METHODS: Medical use data of colorectal cancer patients were obtained from the Korean National Health Insurance Database from 2004 to 2018. We randomly selected $40 \%$ of all colorectal cancer patients $(n=148,848)$. Cognitive impairment was defined as having 1 or more International Classification of Diseases, 10th revision diagnostic codes for dementia or mild cognitive impairment. Patients aged 18 years or younger, patients diagnosed with cognitive impairment before colorectal cancer diagnosis $(n=8,225)$, and patients who did not receive primary resection $(n=45,320)$ were excluded. The effects of individual chemotherapy regimens on cognitive impairment were estimated. We additionally estimated the effect of radiotherapy in rectal cancer patients. Time-dependent competing risk Cox regression was conducted to estimate the overall and age-specific hazard ratios (HR) separately for colon and rectal cancer. Landmark analyses with different lag times were conducted as sensitivity analyses.
\end{abstract}

RESULTS: Chemotherapy did not increase the risk of cognitive impairment in colorectal cancer patients (colon cancer: HR, 0.92 ; $95 \%$ confidence interval [CI], 0.83 to 1.03 ; rectal cancer: $\mathrm{HR}, 0.88 ; 95 \% \mathrm{CI}, 0.75$ to 1.04 ), while radiotherapy was negatively associated with cognitive impairment in rectal cancer patients (HR, $0.01 ; 95 \% \mathrm{CI}, 0.84$ to 0.99 ). Varying directions of the associations between regimens and cognitive impairment were detected. The adverse effect of certain chemotherapy regimens on cognition was more prominent in older adults.

CONCLUSIONS: Chemotherapy and radiotherapy did not increase the risk of cognitive impairment. Older patients with low cognitive reserve could be affected by the adverse cognitive effects of chemotherapy.

KEY WORDS: Chemotherapy, Radiotherapy, Cognitive impairment, Colorectal neoplasms

\section{Correspondence: Sun Jae Jung}

Department of Preventive Medicine, Yonsei University College of Medicine, 50-1 Yonsei-ro, Seodaemun-gu, Seoul 03722, Korea

E-mail: sunjaejung@yuhs.ac

Received: Aug 17, 2021 / Accepted: Nov 2, 2021 / Published: Nov 2, 2021

This article is available from: https://e-epih.org/

(c) This is an open-access article distributed under the terms of the Creative Commons Attribution License (https://creativecommons.org/licenses/by/4.0/), which permits unrestricted use, distribution, and reproduction in any medium, provided the original work is properly cited.

(C) 2021, Korean Society of Epidemiology

\section{INTRODUCTION}

Cancer treatment with chemotherapy and radiotherapy has continually drawn concern regarding its association with cognitive impairment. Although cognitive impairment after chemotherapy, known as "chemo-brain," has attracted considerable attention among researchers, it remains incompletely understood [1-3]. Chemo-brain was first identified and studied in patients with breast cancer who underwent chemotherapy in the 1980s [2], and although some studies have reported potential adverse cognitive effects of chemotherapy [2], a meta-analysis of studies on breast cancer survivors in 2017 reported no overall association 
[1]. Research on chemo-brain in colorectal cancer, however, is relatively sparse. A single-arm study that enrolled about 80 Spanish colorectal cancer patients reported a $50 \%$ increased incidence of cognitive decline. In this study, patients received an oxaliplatin/ fluorouracil regimen [4]. However, a subsequent prospective study in the United States including 362 colorectal cancer patients reported that chemotherapy did not increase the risk of cognitive impairment in cancer patients [5].

In regards to radiotherapy, fewer studies have assessed post-radiotherapy cognitive impairment-or "radio-brain" - in colorectal cancer patients. Two studies conducted in Northern Europe suggested that radiotherapy induces cognitive impairment in rectal cancer patients [6,7]. However, a Swiss study of 60 patients indicated that there was no increased risk of cognitive impairment after radiotherapy [8]. Therefore, evidence for radio-brain in rectal cancer patients is, as of yet, inconclusive, and further in-depth studies of a more extensive population are required.

It has been hypothesized that older patients with cancer are vulnerable to cognitive impairment after chemotherapy [9], as their cognitive reserve-the capacity of the brain to sustain external and internal neuropathological burdens [10] - is diminished [11]. A meta-regression from our previous systematic review and meta-analysis on cognitive decline after chemotherapy in colorectal cancer patients suggested that older colorectal cancer patients are more likely to suffer from cognitive impairment after receiving chemotherapy [12], further supporting this hypothesis. However, the studies that we previously reviewed utilized relatively small and selected populations $(\mathrm{n}<500)$. To support our results, epidemiological evidence from a nationwide, representative study with a large sample is needed.

In this study, we aimed to evaluate the adverse cognitive effects of cancer treatment by conducting a longitudinal analysis of a representative population of Korea. Additionally, we investigated age heterogeneity in the effects of chemotherapy and radiotherapy in patients with colorectal cancer.

\section{MATERIALS AND METHODS}

\section{Participant selection}

Administrative data for medical service usage among colorectal cancer patients were obtained from the Korea National Health Information Database (NHID) from January 1, 2002 to December 31,2018 . The NHID is a public database on healthcare services maintained by the National Health Insurance System (NHIS) of Korea, which is a universal health insurance system that covers the medical expenditures of approximately $98 \%$ of all Korean citizens [13]. The database includes representative and comprehensive information on medical use among Korean patients, including insurance eligibility, diagnostic codes, prescribed medications and procedures, and billing records [14].

Patients with 2 or more International Classification of Diseases, 10th revision (ICD-10) diagnostic codes for colorectal cancer (C18-C20) and 1 or more admission records between 2004 and 2018 were defined as colorectal cancer patients. In order to exclude prevalent cases who had been diagnosed before NHID follow-up and to include incident cases only, we set a washout period of 2 years and excluded patients diagnosed in 2002-2003 [15]. From the original database, $40 \%$ of colorectal cancer patients were randomly selected $(n=148,929)$. We excluded patients aged 18 years or younger $(n=81)$, those diagnosed with cognitive impairment before colorectal cancer diagnosis $(n=8,225)$, and those without administrative records for tumor resection $(n=45,320)$ (Figure 1). In total, 95,303 patients were included in the final analysis $(66,733$ colon cancer cases and 28,570 rectal cancer cases).

\section{Assessing colorectal cancer treatment and cognitive impairment}

Cognitive impairment was defined as the presence of at least 1 ICD-10 diagnostic code for dementia or minor cognitive impairment $[16,17]$. The ICD-10 codes for dementia and minor cognitive impairments are listed in Supplementary Material 1. The claim codes for the cancer treatment modality, including surgical

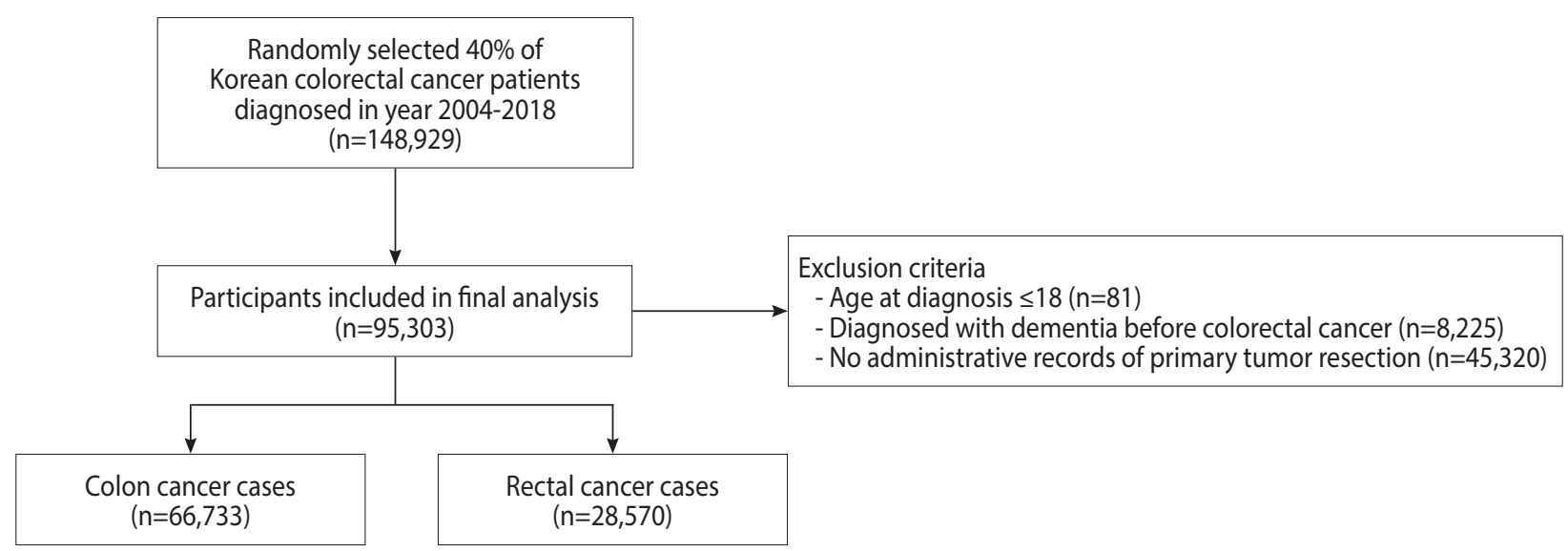

Figure 1. Flow chart of participant inclusion and exclusion. 
resection, chemotherapy, and radiotherapy, were reviewed and confirmed by a colorectal surgeon (CWK), a medical oncologist (HK), and 2 epidemiologists (SJJ, KK). For chemotherapy, regimens that are recommended for first-line chemotherapy in the National Comprehensive Cancer Network (NCCN) Guidelines 2019 were included for analysis (oxaliplatin, capecitabine, 5-fluorouracil [5-FU], irinotecan; the administrative codes are listed in Supplementary Material 2). Patients with at least 1 label for a chemotherapy regimen were considered to be chemotherapy recipients. The date of the first insurance claim for colorectal cancer was considered as the date of colorectal cancer onset.

\section{Covariates}

Monthly insurance premiums were used as a proxy variable for socioeconomic status. The participants' monthly insurance premium payment records at baseline were collected, and the participants were divided into subgroups according to quintile values of monthly insurance premiums (cut-offs: 18,700, 31,110, 44,169, and 63,749 Korean won/mo in 2004). Medical Aid recipients who did not pay a premium due to their poor economic situation were classified into a separate subgroup. The Charlson comorbidity index values at baseline were calculated to assess medical comorbidities [18]. Participants with ICD-10 diagnostic codes corresponding to individual comorbidities were considered to have those comorbidities (Supplementary Material 3).

\section{Statistical analysis}

We classified participants according to the cancer treatment modality in accordance with claim records for cancer treatment as follows: in colon cancer, (1) primary resection only and (2) primary resection with chemotherapy; in rectal cancer, (1) primary resection only, (2) primary resection with chemotherapy, (3) primary resection and radiotherapy, and (4) primary resection with concurrent chemoradiotherapy (CCRT). Using these categories, we described the baseline characteristics of the participants by presenting mean and standard deviation values for continuous variables and numbers and percentages of participants for discrete variables.

We hypothesized that treatments were provided according to the 2019 NCCN guidelines for colorectal cancer treatment and that participants with the same treatment modality were likely to have a similar tumor burden $[19,20]$. To control for possible confounding by tumor burden, we excluded patients without claim codes for surgical resection, thereby excluding patients with inoperable tumors (reflecting a higher tumor burden) and chronic patients receiving palliative treatment only.

Hazard ratios (HRs) and their 95\% confidence intervals (CIs) for chemotherapy and radiotherapy were estimated using a timedependent competing risk survival analysis model, with chemotherapy and radiotherapy being considered as time-dependent variables and all-cause mortality considered as a competing risk [21-24]. To avoid immortal time bias, chemotherapy and radiotherapy were constructed as time-dependent variables, and fol- low-up periods were classified as primary resection with chemotherapy/radiotherapy/CCRT only after patients received the corresponding treatments [25-27]. For instance, if a patient was diagnosed with cognitive impairment after surgical resection and before chemotherapy, the follow-up period of the patient was classified as surgical resection only. Cox regression was conducted by applying the 'proc phreg' procedure in SAS version 9.4 (SAS Institute Inc., Cary, NC, USA). Time-dependent variables were created by 'if else' statements in the 'proc phreg' procedure. Censoring, the event (cognitive impairment), and the competing risk (allcause mortality) were coded as 0,1 , and 2 respectively (Supplementary Material 4). We also estimated HRs for chemotherapy regimen combinations for colorectal cancer treatment, including FOLFOX (folate, 5-FU, and oxaliplatin), FOLFIRI (folate, 5-FU, and irinotecan), FOLFOXIRI (folate, 5-FU, oxaliplatin, and irinotecan), CapeOx (capecitabine and oxaliplatin), capecitabine only, 5-FU only, and irinotecan only. Considering left truncation, the date of colorectal cancer diagnosis was used as the date of follow-up initiation. Age interaction terms were added to the model to assess the moderating effects of age, and conditional HRs of chemotherapy and radiotherapy by age points were estimated [28]. All models were adjusted for age, sex, comorbidities, and the monthly insurance premium.

For sensitivity analyses, we redefined cognitive impairment cases as patients with 2 or more corresponding diagnostic codes for cognitive impairment and repeated the survival analyses. Additionally, we performed landmark analyses by conducting timefixed Cox regression with lag times of 6 months, 12 months, and 18 months [29]. All statistical analyses were conducted using SAS version 9.4 .

\section{Ethics statement}

The study protocols were approved by the Institutional Review Board of Yonsei University Health System, Seoul, Korea (approval No. 4-2019-0425). Informed consent was waived for this study, since personal information that can be used to identify individuals registered to NHID was removed. All procedures contributing to this work complied with the ethical standards of the relevant national and institutional committees on human experimentation and with the 1975 Declaration of Helsinki, which was revised in 2008.

\section{RESULTS}

\section{Characteristics of the study population}

Among the 66,733 patients with colon cancer at baseline (2004), 14,146 (21.2\%) received adjuvant or neoadjuvant chemotherapy until 2018. The mean follow-up duration was longer in patients who did not receive chemotherapy than in those who received it (5.57 vs. 3.21 years, $\mathrm{p}<0.001)$. Capecitabine, oxaliplatin, 5-FU, and irinotecan were administered to 3,228 (22.8\%), 9,928 (70.2\%), 7,709 (54.5\%), and 2,444 (17.3\%) chemotherapy recipients, respectively. The incidence rates of cognitive impairment 
were 22.17 per 1,000 person-years in chemotherapy non-recipients and 14.48 per 1,000 person-years in chemotherapy recipients. The all-cause mortality rates were 49.05 per 1,000 personyears in chemotherapy non-recipients and 96.52 per 1,000 person-years in patients who received chemotherapy (Table 1).

Among the 28,570 patients with rectal cancer included in the analyses, 2,604 (9.1\%) received chemotherapy, 8,098 (28.3\%) received radiotherapy, and 3,161 (11.1\%) received CCRT before or after surgical resection. The mean follow-up duration (in years) was relatively longer in the primary resection and resection-radiotherapy combination groups and shorter in the resection-chemotherapy combination and resection-CCRT combination groups. Among 5,765 chemotherapy and CCRT recipients, capecitabine, oxaliplatin, 5-FU, and irinotecan were administered to 1,734 (30.1\%), 3,289 (57.0\%), 3,499 (60.7\%), and 1,228 (21.3\%), respectively. The incidence rate of cognitive impairment was highest in the resection only group (23.16 per 1,000 person-years), while mortality was highest in the resection-CCRT combination group (123.66 per 1,000 person-years) (Table 1).

\section{Effects of chemotherapy and radiotherapy on cognitive impairment}

In colon cancer patients, chemotherapy did not increase the risk of cognitive impairment (HR, 0.92; 95\% CI, 0.83 to 1.03). In rectal cancer patients, neither chemotherapy (HR, 0.88; 95\% CI, 0.75 to 1.04 ) nor radiotherapy (HR, $0.91 ; 95 \% \mathrm{CI}, 0.84$ to 0.99 ) was positively associated with cognitive impairment. Folate administration during chemotherapy was negatively associated with cognitive impairment in both colon cancer (HR, 0.66; 95\% CI, 0.45 to 0.97 ) and rectal cancer (HR, $0.52 ; 95 \% \mathrm{CI}, 0.31$ to 0.88 ) (Table 2). The age-specific HRs of chemotherapy and radiotherapy were larger in older patients, but were not significant in any age spectra. The protective effect of folate administration was more prominent in older adult patients (Figure 2).

When analyzed by regimen, the FOLFOX regimen was negatively associated with cognitive impairment in both colon cancer (HR, 0.44; 95\% CI, 0.32 to 0.60 ) and rectal cancer (HR, 0.53; 95\% CI, 0.34 to 0.82). The FOLFOXIRI regimen showed lower HRs in rectal cancer (HR, $0.49 ; 95 \% \mathrm{CI}, 0.26$ to 0.91 ), but not in colon cancer (HR, $0.85 ; 95 \% \mathrm{CI}, 0.58$ to 1.27 ). In general, patients who received the CapeOx or capecitabine-only regimen showed increased hazards for cognitive impairment, although the magnitude of the association varied by primary cancer site (Table 2). The age-specific HRs for FOLFIRI and FOLFOXIRI were higher in older patients than in younger patients, although the HRs were non-significant in all age spectra. The direction of the interaction between the effects of the CapeOx regimens and age was positive in colon cancer, but negative in rectal cancer. The capecitabineonly regimen and radiotherapy did not show significant interactions with age (Figure 3 ).

The sensitivity analysis showed similar results to those of the main analysis. When cognitive impairment was redefined as having 2 or more corresponding ICD-10 codes, the estimated HRs of

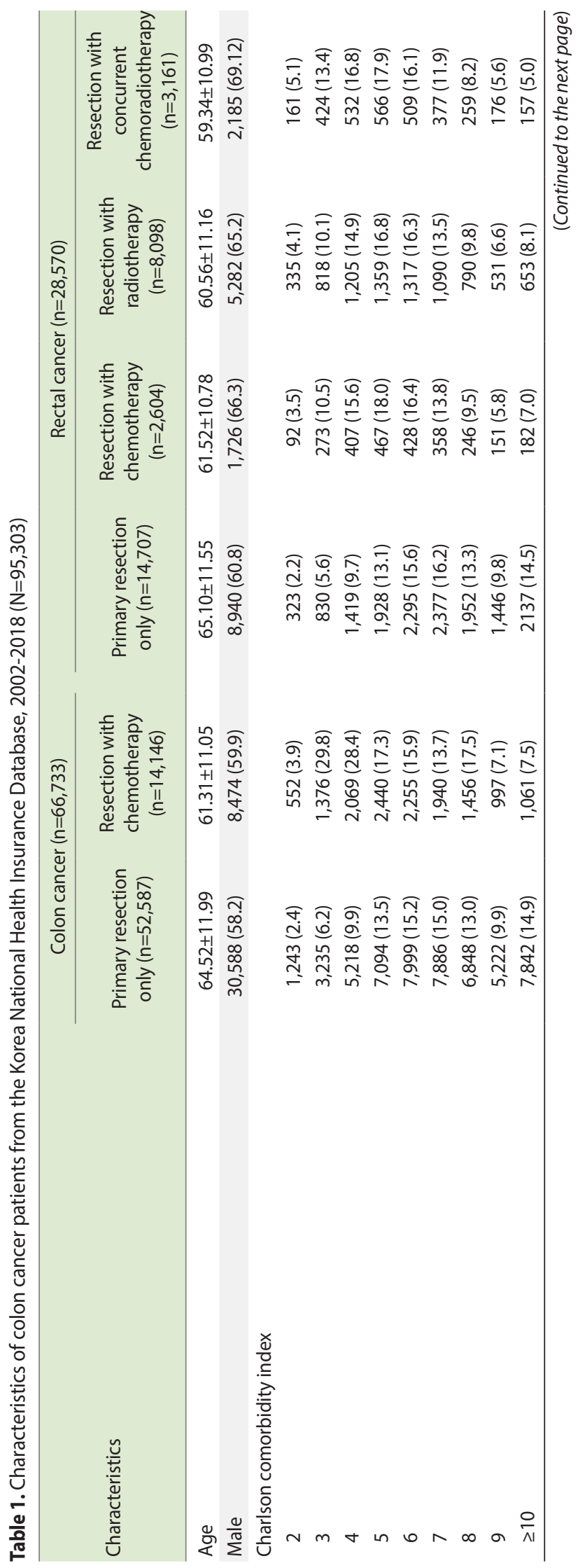




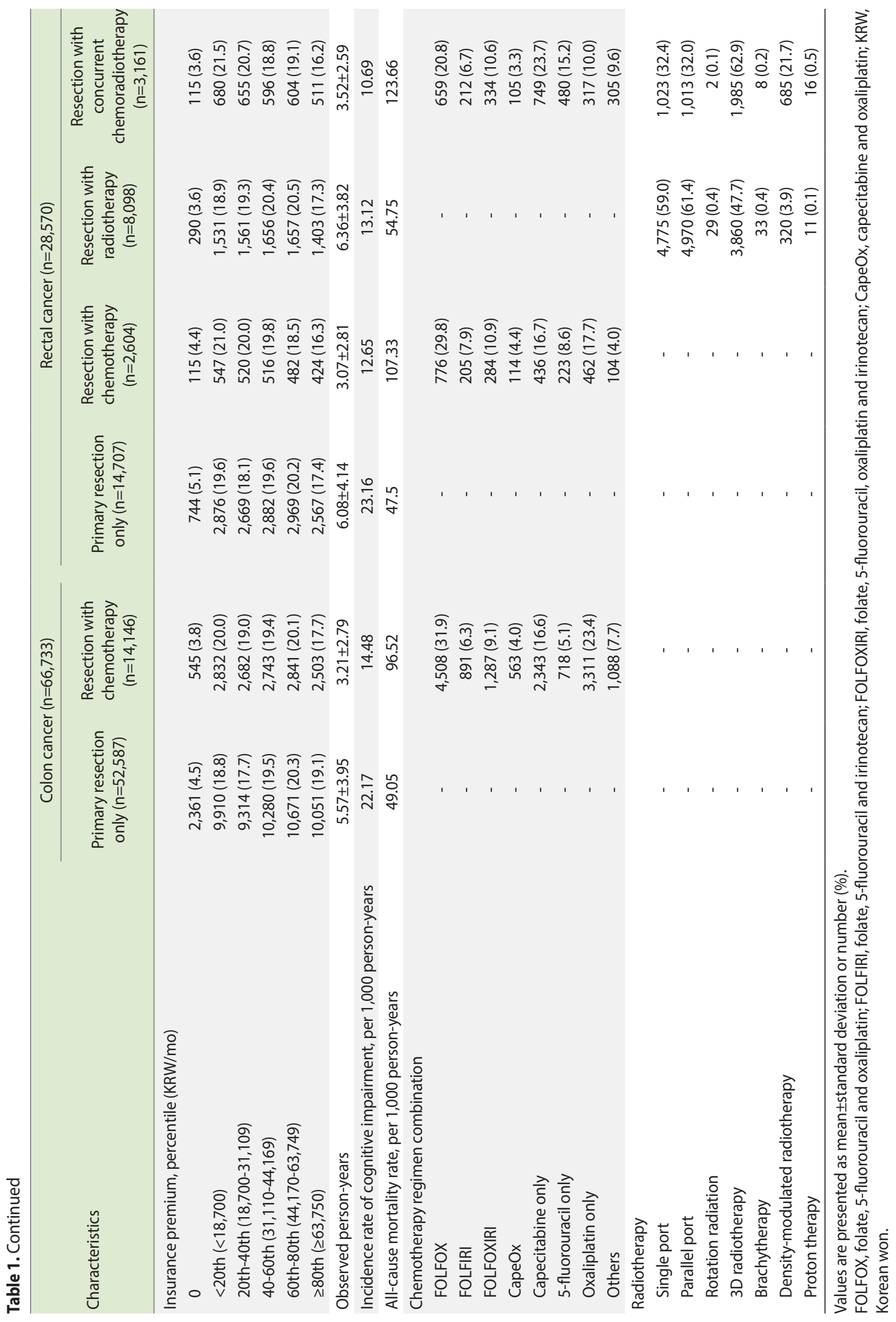


Table 2. Estimated hazard ratios of chemotherapy regimens and radiotherapy on cognitive impairment $(n=95,303)^{1}$

\begin{tabular}{lcc}
\hline Variables & Colon cancer $(n=66,733)$ & Rectal cancer $(n=28,570)$ \\
\hline Surgical resection only & 1.00 (reference) & $1.00($ reference $)$ \\
Chemotherapy, overall & $0.92(0.83,1.03)$ & $0.88(0.75,1.04)$ \\
Folate therapy, overall & $0.66(0.45,0.97)$ & $0.52(0.31,0.88)$ \\
Chemotherapy, by regimen & & $0.53(0.34,0.82)$ \\
FOLFOX & $0.44(0.32,0.60)$ & $1.43(0.87,2.35)$ \\
FOLFIRI & $1.22(0.77,1.94)$ & $0.49(0.26,0.91)$ \\
FOLFOXIRI & $0.85(0.58,1.27)$ & $1.60(0.93,2.76)$ \\
CapeOx & $1.33(0.82,2.18)$ & $1.01(0.76,1.34)$ \\
Capecitabine only & $1.37(1.16,1.62)$ & $0.71(0.47,1.05)$ \\
5-FU only & $0.86(0.54,1.37)$ & $0.91(0.65,1.29)$ \\
Oxaliplatin only & $0.73(0.60,0.89)$ & \\
\hline
\end{tabular}

Values are presented as hazard ratio (95\% confidence interval).

Overall, chemotherapy and radiotherapy were not significantly associated with cognitive impairment in colorectal cancer. Folate therapy was negatively associated with cognitive impairment. When analyzed by regimen, FOLFOX and FOLFOXIRI decreased the risk of cognitive impairment, while the CapeOx and capecitabine-only regimens were positively associated with cognitive impairment.

FOLFOX, folate, 5-fluorouracil (5-FU). oxaliplatin; FOLFIRI, folate, 5-FU, irinotecan; FOLFOXIRI, folate, 5-FU, oxaliplatin, irinotecan; CapeOx: capecitabine, oxaliplatin.

'All models are adjusted for age, sex, Charlson comorbidity index, and monthly insurance premium.
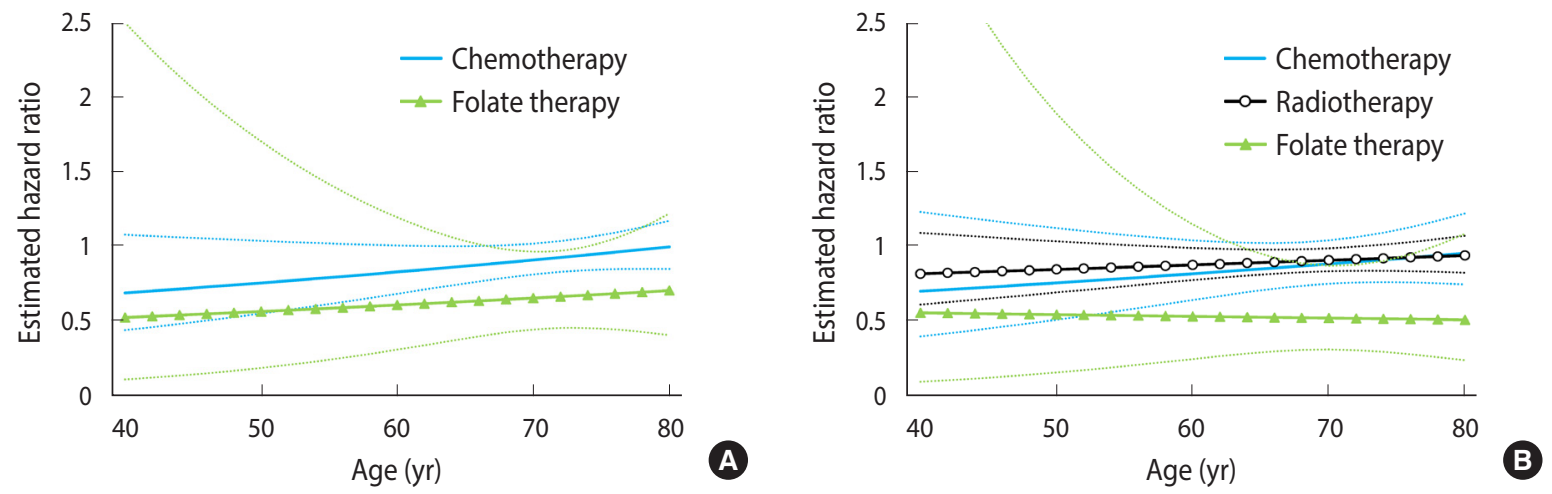

Figure 2. Age-specific hazard ratios of chemotherapy and radiotherapy for cognitive impairment (A) colon cancer and (B) rectal cancer.
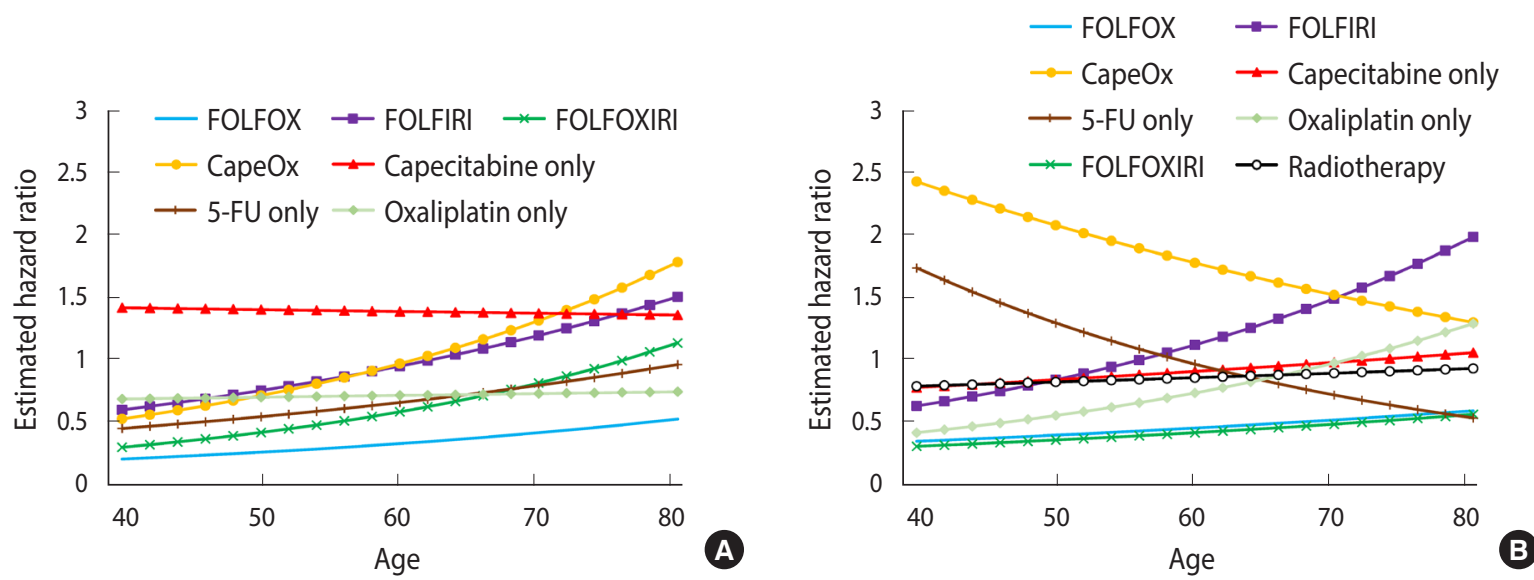

Figure 3. Age-specific hazard ratios of chemotherapy regimen and radiotherapy for cognitive impairment (A) colon cancer and (B) rectal cancer. FOLFOX, folate, 5-fluorouracil (5-FU), oxaliplatin; FOLFIRI, folate, 5-FU, irinotecan; FOLFOXIRI, folate, 5-FU, oxaliplatin, irinotecan; CapeOx: capecit abine, oxaliplatin. 
chemotherapy and radiotherapy did not show significant differences from the main analyses (Supplementary Material 5). In landmark analyses, as the time lag increased, the estimated HRs of chemotherapy decreased and protective effects of folate therapy became more prominent (Supplemental Material 6). The interaction trends detected in landmark analyses were similar to those of the main analyses (Supplemental Material 7). Landmark analyses by regimen showed similar trends to those of the main analyses (Supplemental Material 8).

\section{DISCUSSION}

Overall, chemotherapy and radiotherapy did not increase the risk of cognitive impairment in colorectal cancer. While the CapeOx and capecitabine-only regimens increased the risk, the FOLFOX and oxaliplatin-only regimens were negatively associated with cognitive impairment. The FOLFORI regimen was likely to be beneficial in younger patients, but increased the risk of cognitive impairment in older patients. Radiotherapy was not associated with an increased risk of cognitive impairment.

The findings of our analyses imply that the characteristics of the primary tumor, both biological and psychosocial, play an important role in the manifestation of chemotherapy-related cognitive impairment. It is well known that a significant proportion of breast cancer patients suffer from depression and anxiety, which lead to dysfunctional cognition and general fatigue [30]. A recent meta-analysis reported that around $32 \%$ of breast cancer patients suffer from depression [31]. In contrast, the results from a systematic review on depression and anxiety in colorectal cancer patients reported that only around $6 \%$ of colorectal cancer patients are affected by depression [32]. These differences in the psychological consequences of tumors, alongside variance in the biological action of chemotherapeutic agents [33,34], could have resulted in the varying directions of associations in our study. A largescale randomized clinical trial on colorectal cancer patients and further research on the mechanisms of chemotherapy-related cognitive impairment are warranted to achieve a better understanding of these phenomena.

Adverse effects of chemotherapy appear to be more likely in older patients. Cognitive reserve, which reflects the capacity of the brain to withstand the effects of external events, toxins, or diseases that can affect cognitive function [10], is known to be associated with the vulnerability of the brain to the neurotoxicity of chemotherapeutic agents [35]. It has been postulated that cancer treatments interact with aging of the brain and accelerate cognitive decline, as brain images of cancer treatment receivers showed structural changes in the brain that were indicative of aging [36,37]. However, since several chemotherapeutic agents did not increase the risk of cognitive impairment even in older patients, care must be taken when interpreting our results.

Our study provides evidence of adverse cognitive effects of cancer treatment in colorectal patients from real-world data. Our results from a representative population of Korea suggest heteroge- neity according to age in cognitive decline among colorectal cancer patients after treatment. This is one of only a few studies to utilize nationwide data in an attempt to investigate chemotherapy-related and radiotherapy-related cognitive dysfunction in colorectal cancer patients. However, this study does have some limitations. First, several chemotherapeutic agents are not covered by the NHIS, which might have caused selection bias; in particular, most non-covered regimens are for second-line treatment or palliative treatment in advanced cancer. To minimize selection bias, we excluded patients without primary cancer resection records, which could include substantial missing values.

Second, due to administrative challenges in obtaining medical records, the validity of the date of cognitive impairment onset might be questioned. As the data were collected for medical insurance administration and not for research, information on disease and mortality might have been misclassified [14]. Additionally, there might be concerns about the adequacy of assessment and treatment for cognitive impairment. During the process of cancer treatment, it is likely that patients and physicians are more focused on controlling neoplasms than on controlling complications. However, since chemo-brain is a well-known complication that is widely acknowledged by physicians and cancer survivors $[3,38]$, it is unlikely that cognitive impairment after cancer treatment would be left uncontrolled. Additionally, the reliability of cognitive impairment diagnosis in the Korean NHID has been validated [39].

Lastly, due to the study design, selective survival bias is possible. Since the mean follow-up time was shorter in chemotherapy or radiotherapy recipients, their chances of developing cognitive impairment would be decreased. To address potential selective survival bias, we applied time-dependent competing risk survival models $[21,22,24,40]$. Evidence from a randomized clinical trial comparing patients with different clinical cancer stages would provide a better understanding of the true associations.

In conclusion, our results from a representative nationwide database of Korea suggest that chemotherapy and radiotherapy do not impose marked adverse cognitive effects in colorectal cancer patients. Our study provides evidence that contributes to a better understanding of the nature of cancer treatment-related cognitive impairment in colorectal cancer patients. A large-scale randomized clinical trial with a longer follow-up period is needed to thoroughly investigate the complex mechanisms of adverse effects in cancer treatment. Regular follow-up assessing cognitive function after cancer treatment could help prevent cognitive impairments in older patients with low cognitive reserve.

\section{SUPPLEMENTARY MATERIALS}

Supplementary materials are available at http://www.e-epih.org/.

\section{CONFLICT OF INTEREST}

The authors have no conflicts of interest to declare for this study. 


\section{FUNDING}

This study was supported by a National Research Foundation of Korea grant funded by the Ministry of Science and ICT (grant number 2020R1C1C1003502).

\section{ACKNOWLEDGEMENTS}

None.

\section{AUTHOR CONTRIBUTIONS}

Conceptualization: KK, SJJ. Data curation: KK, SJJ. Formal analysis: KK. Funding acquisition: SJJ. Methodology: KK, SJJ. Project administration: KK. Visualization: KK, SJJ. Writing original draft: KKH, SJJ. Writing - review \& editing: KK, CWK, AS, HK, SJJ.

\section{ORCID}

Kwanghyun Kim: https://orcid.org/0000-0001-9552-5085; Chang Woo Kim: https://orcid.org/0000-0002-6317-8354; Aesun Shin: https://orcid.org/0000-0002-6426-1969; Hyunseok Kang: https:// orcid.org/0000-0001-5758-8202; Sun Jae Jung: https://orcid.org/ 0000-0002-5194-7339

\section{REFERENCES}

1. Bernstein LJ, McCreath GA, Komeylian Z, Rich JB. Cognitive impairment in breast cancer survivors treated with chemotherapy depends on control group type and cognitive domains assessed: a multilevel meta-analysis. Neurosci Biobehav Rev 2017;83:417-428.

2. Matsuda T, Takayama T, Tashiro M, Nakamura Y, Ohashi Y, Shimozuma K. Mild cognitive impairment after adjuvant chemotherapy in breast cancer patients--evaluation of appropriate research design and methodology to measure symptoms. Breast Cancer 2005;12:279-287.

3. Dietrich J, Prust M, Kaiser J. Chemotherapy, cognitive impairment and hippocampal toxicity. Neuroscience 2015;309:224-232.

4. Cruzado JA, López-Santiago S, Martínez-Marín V, José-Moreno G, Custodio AB, Feliu J. Longitudinal study of cognitive dysfunctions induced by adjuvant chemotherapy in colon cancer patients. Support Care Cancer 2014;22:1815-1823.

5. Vardy JL, Dhillon HM, Pond GR, Rourke SB, Bekele T, Renton C, et al. Cognitive function in patients with colorectal cancer who do and do not receive chemotherapy: a prospective, longitudinal, controlled study. J Clin Oncol 2015;33:4085-4092.

6. Bruheim K, Guren MG, Skovlund E, Hjermstad MJ, Dahl O, Frykholm G, et al. Late side effects and quality of life after radiotherapy for rectal cancer. Int J Radiat Oncol Biol Phys 2010;76: 1005-1011.

7. Couwenberg AM, Burbach JP, van Grevenstein WM, Smits AB,
Consten EC, Schiphorst AH, et al. Effect of neoadjuvant therapy and rectal surgery on health-related quality of life in patients with rectal cancer during the first 2 years after diagnosis. Clin Colorectal Cancer 2018;17:e499-e512.

8. Allal AS, Gervaz P, Gertsch P, Bernier J, Roth AD, Morel P, et al. Assessment of quality of life in patients with rectal cancer treated by preoperative radiotherapy: a longitudinal prospective study. Int J Radiat Oncol Biol Phys 2005;61:1129-1135.

9. Ahles TA, Saykin AJ, McDonald BC, Li Y, Furstenberg CT, Hanscom BS, et al. Longitudinal assessment of cognitive changes associated with adjuvant treatment for breast cancer: impact of age and cognitive reserve. J Clin Oncol 2010;28:4434-4440.

10. Whalley LJ, Deary IJ, Appleton CL, Starr JM. Cognitive reserve and the neurobiology of cognitive aging. Ageing Res Rev 2004;3: 369-382.

11. Hurria A, Rosen C, Hudis C, Zuckerman E, Panageas KS, Lachs MS, et al. Cognitive function of older patients receiving adjuvant chemotherapy for breast cancer: a pilot prospective longitudinal study. J Am Geriatr Soc 2006;54:925-931.

12. Hwang SY, Kim K, Ha B, Lee D, Kim S, Ryu S, et al. Neurocognitive effects of chemotherapy for colorectal cancer: a systematic review and a meta-analysis of 11 studies. Cancer Res Treat 2021; 53:1134-1147.

13. Kwon S. Thirty years of national health insurance in South Korea: lessons for achieving universal health care coverage. Health Policy Plan 2009;24:63-71.

14. Seong SC, Kim YY, Khang YH, Heon Park J, Kang HJ, Lee H, et al. Data resource profile: the National Health Information Database of the National Health Insurance Service in South Korea. Int J Epidemiol 2017;46:799-800.

15. Shin J, Yoon HY, Lee YM, Ha E, Lee JH. Inhaled corticosteroids in COPD and the risk for coronary heart disease: a nationwide cohort study. Sci Rep 2020;10:18973.

16. Suh Y, Ah YM, Han E, Jun K, Hwang S, Choi KH, et al. Dose response relationship of cumulative anticholinergic exposure with incident dementia: validation study of Korean anticholinergic burden scale. BMC Geriatr 2020;20:265.

17. Lee J, Jung SJ, Choi JW, Shin A, Lee YJ. Use of sedative-hypnotics and the risk of Alzheimer's dementia: a retrospective cohort study. PLoS One 2018;13:e0204413.

18. Charlson ME, Pompei P, Ales KL, MacKenzie CR. A new method of classifying prognostic comorbidity in longitudinal studies: development and validation. J Chronic Dis 1987;40:373-383.

19. Benson AB, Venook AP, Al-Hawary MM, Cederquist L, Chen YJ, Ciombor KK, et al. NCCN guidelines insights: colon cancer, version 2.2018. J Natl Compr Canc Netw 2018;16:359-369.

20. Benson AB, Venook AP, Al-Hawary MM, Cederquist L, Chen YJ, Ciombor KK, et al. Rectal cancer, version 2.2018, NCCN clinical practice guidelines in oncology. J Natl Compr Canc Netw 2018;16: 874-901.

21. Latouche A, Porcher R, Chevret S. A note on including time-dependent covariate in regression model for competing risks data. Biom J 2005;47:807-814. 
22. Ning J, Bandeen-Roche K. Estimation of time-dependent association for bivariate failure times in the presence of a competing risk. Biometrics 2014;70:10-20.

23. Fuerst D, Frank S, Mueller C, Beelen DW, Schetelig J, Niederwieser $\mathrm{D}$, et al. Competing-risk outcomes after hematopoietic stem cell transplantation from the perspective of time-dependent effects. Haematologica 2018;103:1527-1534.

24. Ng DK, Antiporta DA, Matheson MB, Muñoz A. Nonparametric assessment of differences between competing risk hazard ratios: application to racial differences in pediatric chronic kidney disease progression. Clin Epidemiol 2020;12:83-93.

25. Lévesque LE, Hanley JA, Kezouh A, Suissa S. Problem of immortal time bias in cohort studies: example using statins for preventing progression of diabetes. BMJ 2010;340:b5087.

26. Suissa S. Immortal time bias in observational studies of drug effects. Pharmacoepidemiol Drug Saf 2007;16:241-249.

27. Austin PC, Latouche A, Fine JP. A review of the use of time-varying covariates in the Fine-Gray subdistribution hazard competing risk regression model. Stat Med 2020;39:103-113.

28. Leona S, Aiken LS, West SG, Reno RR. Multiple regression: testing and interpreting interactions. Newbury Park: SAGE; 1996, p. 31-60.

29. Anderson JR, Cain KC, Gelber RD. Analysis of survival by tumor response. J Clin Oncol 1983;1:710-719.

30. Bower JE. Behavioral symptoms in patients with breast cancer and survivors. J Clin Oncol 2008;26:768-777.

31. Pilevarzadeh M, Amirshahi M, Afsargharehbagh R, Rafiemanesh $\mathrm{H}$, Hashemi SM, Balouchi A. Global prevalence of depression among breast cancer patients: a systematic review and metaanalysis. Breast Cancer Res Treat 2019;176:519-533.

32. Peng YN, Huang ML, Kao CH. Prevalence of depression and anxiety in colorectal cancer patients: a literature review. Int J Environ Res Public Health 2019;16:411.

33. Lange M, Joly F, Vardy J, Ahles T, Dubois M, Tron L, et al. Cancer-related cognitive impairment: an update on state of the art, detection, and management strategies in cancer survivors. Ann Oncol 2019;30:1925-1940.

34. Vardy J, Dhillon HM, Pond GR, Rourke SB, Xu W, Dodd A, et al. Cognitive function and fatigue after diagnosis of colorectal cancer. Ann Oncol 2014;25:2404-2412.

35. Ahles TA. Brain vulnerability to chemotherapy toxicities. Psychooncology 2012;21:1141-1148.

36. Maccormick RE. Possible acceleration of aging by adjuvant chemotherapy: a cause of early onset frailty? Med Hypotheses 2006; 67:212-215.

37. Ahles TA, Root JC, Ryan EL. Cancer- and cancer treatment-associated cognitive change: an update on the state of the science. J Clin Oncol 2012;30:3675-3686.

38. Janelsins MC, Kesler SR, Ahles TA, Morrow GR. Prevalence, mechanisms, and management of cancer-related cognitive impairment. Int Rev Psychiatry 2014;26:102-113.

39. Kang J, Shin DW, Han K, Park SH, Lee WG, Yoo JE, et al. Risk of dementia in prostate cancer survivors: a nationwide cohort study in Korea. Curr Probl Cancer 2020;44:100578.

40. Kleinbaum DG, Klein M. Survival analysis. New York: Springer; 2010, p. 426-451. 